\title{
EFEITO DA ESTAQUIA, MINIESTAQUIA, MICROESTAQUiA E MICROPROPAGAÇÃO NO DESEMPENHO SILVICULTURAL DE CLONES HÍBRIDOS DE Eucalyptus spp. ${ }^{1}$
}

Marcelo Lelis de Oliveira ${ }^{2}$, Aloisio Xavier ${ }^{3}$, Alex Passos dos Santos ${ }^{4}$ e Helder Bolognani Andrade ${ }^{5}$

\begin{abstract}
RESUMO - Este trabalho objetivou avaliar a “performance” de quatro clones híbridos de Eucalyptus spp., propagados pelas técnicas de estaquia, micropropagação, microestaquia e miniestaquia, quanto às características de crescimento em altura e diâmetro (dap), bem como a produção de biomassa da parte aérea, em teste clonal, instalado em dois locais distintos localizados no norte de Minas Gerais. De acordo com os resultados, podese concluir que os efeitos das técnicas de propagação não resultaram em diferenças expressivas no crescimento em altura e dap, nas avaliações aos 4, 8, 16 e 24 meses de idade, dos clones estudados. Em relação à biomassa da parte aérea, os resultados não indicaram uma tendência sobre possíveis efeitos das técnicas de propagação em relação ao comportamento silvicultural dos clones.
\end{abstract}

Palavras-chave: Silvicultura clonal, propagação vegetativa e clonagem.

\section{EFFECT OF CUTTING, MINICUTTING, MICROCUTTING AND MICROPROPAGATION ON THE SILVICULTURAL PERFORMANCE OF Eucalyptus spp. HYBRID CLONES}

\begin{abstract}
The objective of the present study was to evaluate the performance of four Eucalyptus spp. hybrid clones, propagated by cutting, micropopagation, microcutting and minicutting techniques. The study evaluated height and diameter growth characteristics, as well as the aerial biomass production, in a clonal test installed in two localities in the north of Minas Gerais State. According to the results, it can be concluded that the effects of propagation techniques did not result in expressive differences in height growth and DBH, at ages 4, 8, 16 and 24 months, for the studied clones. In relation to the aerial biomass, the results did not indicate a tendency for possible effects of the propagation techniques on clone silvicultural performance.
\end{abstract}

Keywords: Clonal forestry, vegetative propagation and cloning.

\section{INTRODUÇÃO}

A silvicultura clonal tem como ponto de partida a seleção de genótipos superiores para, posteriormente, proceder-se à propagação clonal massal. No entanto, para comprovar a superioridade genética dos indivíduos fenotipicamente selecionados é necessário que eles sejam multiplicados e avaliados em testes clonais. Contudo, experiências com algumas espécies têm mostrado que propágulos vegetativos utilizados na propagação clonal não representam fielmente o crescimento e desenvolvimento das árvores selecionadas, podendo resultar em vários graus de redução nos

\footnotetext{
${ }^{1}$ Recebido em 13.02.2004 e aceito para publicação em 05.04.2006.

${ }^{2}$ KLABIN S.A.

${ }_{3}^{3}$ Departamento de Engenharia Florestal da Universidade Federal de Viçosa, 36570-000, Viçosa-MG. E-mail: <xavier@ufv.br>. ${ }^{4}$ RIPASA S.A

${ }^{5} \mathrm{~V} \&$ M Florestal.
} 
potenciais benefícios da clonagem, como a diminuição de produtividade (FRAMPTON e FOSTER, 1993).

Nesse caso, um dos fatores em potencial que interferem no crescimento das árvores e pode induzir a erros no processo de seleção está relacionado com as mudanças morfológicas e fisiológicas que as plantas arbóreas sofrem durante o seu ciclo de vida. Assim, propágulos originados de determinadas partes da planta, que propiciam menor crescimento, podem levar à rejeição de clones com elevado valor genético.

Portanto, a escolha dos propágulos vegetativos ideais visa diminuir perdas relacionadas à não-aptidão para a propagação vegetativa, formar mudas com alto vigor da parte aérea e radicular e também evitar o declínio do crescimento no campo, entre outras características. Segundo Hackett e Murray (1993), o conhecimento das mudanças que ocorrem durante o ciclo de vida de algumas plantas arbóreas tem importância prática significativa, pois a produtividade e a qualidade de uma espécie florestal estão correlacionadas com o seu grau de maturidade. Assim, a compreensão dos aspectos relacionados com as mudanças morfológicas e fisiológicas dos propágulos vegetativos utilizados na propagação e de seu posterior desenvolvimento no campo irá incrementar, em muito, o sucesso dos programas de silvicultura clonal.

Nessa linha de pesquisa, Xavier e Comério (1996) relataram que a heterogeneidade entre plantas em plantios comerciais de um mesmo clone é provocada pelo efeito "C" (efeito da clonagem) e pode ser minimizada com a utilização da micropropagação. Bell et al. (1993), Denison e Kietzka (1993) e Rockwood e Warrag (1994) afirmaram que o processo da micropropagação produz maior uniformidade e produtividade no plantio de um mesmo clone em relação à macropropagação. Essa maior uniformidade e produtividade das árvores pela micropropagação, segundo Xavier et al. (1997) são provenientes da utilização de material vegetativo rejuvenescido in vitro, que promove a minimização do efeito "C". Embora a influência do efeito "C" possa ser reduzida, pesquisas têm evidenciado que se deve tomar precaução na produção de propágulos destinados à propagação num programa de implantação de testes clonais (FRAMPTON e FOSTER, 1993).

Atualmente, o uso das técnicas de microestaquia e miniestaquia pela maioria das empresas do setor florestal brasileiro representa a aplicação de conhecimentos relacionados ao gradiente de maturação e do rejuvenescimento no processo de produção de mudas de Eucalyptus, em que ganhos em fase de viveiro com a utilização de propágulos mais juvenis estão bem evidenciados (XAVIER e COMÉRIO, 1996; ASSIS, 1997; XAVIER et al., 2001; TITON, 2001). Basicamente a microestaquia diferencia-se da miniestaquia pela origem do material que compõe o jardim clonal, aonde na microestaquia as cepas originam-se de mudas micropropagadas e na miniestaquia, as cepas iniciais são formadas de mudas propagadas pela estaquia convencional. Assim, espera-se que a microestaquia apresente melhor performance na propagação clonal em relação a miniestaquia, visto o rejuvenescimento promovido previamente por meio da micropropagação.

Ganhos em crescimento no campo com o uso de propágulos vegetativos mais juvenis com o uso da micropropagação e possíveis ganhos com a microestaquia em comparação com a técnica de estaquia foram citados por Xavier et al. (1997). No entanto, a implantação de teste clonal no campo com uso de propágulos vegetativos com diferentes graus de juvenilidade, como a estaquia, micropropagação, microestaquia e miniestaquia, ainda é pouco conhecida. Um melhor entendimento dos efeitos do gradiente de juvenilidade e do rejuvenescimento em clones de Eucalyptus selecionados em idade adulta permitirá uma melhor interpretação dos testes clonais e uma redução de possíveis efeitos, que afetam a repetibilidade de um determinado clone, além da confirmação dos possíveis ganhos obtidos com o uso de propágulos mais juvenis.

Resultados de campo também podem contribuir muito para a tomada de decisão sobre o uso de uma determinada técnica em detrimento de outra, visando a confirmação da melhor estratégia a ser adotada, pois em condições de viveiro a técnica de microestaquia mostrou-se superior à técnica de miniestaquia (TITON, 2001; XAVIER et al., 2001), principalmente no que tange a clones de baixo enraizamento. Por outro lado, existem clones com grande facilidade de propagação vegetativa, em que procedimentos mais simples e de menor custo, como a estaquia e miniestaquia, podem ser uma alternativa viável (XAVIER et al., 2001).

Desta forma, o presente trabalho objetivou avaliar o desempenho de quatro clones de Eucalyptus spp., propagados pelas técnicas de estaquia, micropropagação, microestaquia e miniestaquia, quanto às características 
de crescimento em altura e diâmetro (dap), bem como a produção de biomassa da parte aérea em condições de campo, em dois sítios diferentes localizados na região norte de Minas Gerais.

\section{MATERIAL E MÉTODOS}

No presente estudo, os efeitos da propagação clonal por estaquia, micropropagação, microestaquia e miniestaquia, quanto ao desempenho no campo de 4 clones de Eucalyptus, foram avaliados em dois testes clonais e em duas parcelas experimentais instalados em dois locais distintos: o primeiro localizado em Bocaiúva, MG, na Fazenda Corredor (Local 1), com precipitação média anual de $931 \mathrm{~mm}$ e solo de textura predominantemente argilosa e o segundo, localizado no Município de João Pinheiro, MG, na Fazenda Campo Alegre (Local 2), apresentando precipitação média anual de $1.189 \mathrm{~mm}$ e solo de textura predominantemente arenosa.

Os materiais genéticos utilizados foram quatro clones de Eucalyptus spp.: sendo um clone de Eucalyptus tereticornis $x$ Eucalyptus pellita (VM1), dois clones híbridos de Eucalyptus grandis x Eucalyptus sp. (VM2 e VM3) e um clone de Eucalyptus urophylla $x$ Eucalyptus grandis (VM4). As mudas utilizadas na instalação dos testes clonais e das parcelas experimentais foram produzidas a partir de jardins clonais instalados no Viveiro Florestal da V \& M Florestal. As mudas foram obtidas por meio da propagação vegetativa, pelas técnicas de estaquia, micropropagação, microestaquia e miniestaquia, de acordo com os procedimentos descritos nos itens subseqüentes.

\subsection{Obtenção das mudas clonais}

As mudas obtidas pela técnica de estaquia foram produzidas a partir de propágulos vegetativos (estacas), variando de 8 a $10 \mathrm{~cm}$ de comprimento, oriundas de jardim clonal instalado em área de campo. Essas estacas foram enraizadas em casa de vegetação (permanência de 30 dias), com a aplicação, na base das estacas, do regulador de crescimento ácido indolbutírico (AIB) na concentração de $4.000 \mathrm{mg} \mathrm{L}^{-1}$. Posteriormente, as estacas enraizadas foram aclimatadas em casa de sombra, com sombrite $50 \%$ (permanência de 10 dias) e rustificadas a pleno sol até aos 90 dias de idade.

A produção das mudas referente à técnica de microestaquia foi obtida pelo enraizamento de microestacas, com dimensões variando de 4 a $6 \mathrm{~cm}$ de comprimento, coletadas no jardim microclonal, localizado em pleno sol. O enraizamento ocorreu em casa de vegetação, com a permanência de 25 dias. Posteriormente, as microestacas foram transferidas para casa de sombra, com sombrite $50 \%$, onde permaneceram oito dias para aclimatação. Finalmente foram para pleno sol até completarem 75 dias de idade. Na técnica de miniestaquia, as mudas foram produzidas pelo enraizamento de miniestacas, coletadas no jardim miniclonal formado a partir de mudas advindas da técnica de estaquia, tendo as demais etapas seguido os mesmos procedimentos da técnica de microestaquia.

As mudas micropropagadas foram obtidas após a micropropagação a partir da proliferação de gemas axilares. Os clones passaram por 10 a 12 subcultivos em meio de cultura adequado, com a finalidade de obter o rejuvenescimento. Após o alongamento das gemas in vitro, estas foram transplantadas em casa de vegetação para o enraizamento ex vitro, em tubetes plásticos de $55 \mathrm{~cm}^{3}$ de capacidade contendo substrato constituído de partes iguais de vermiculita de granulometria média e casca de arroz carbonizada. Essa fase foi realizada no Laboratório de Cultura de Tecidos II do Instituto de Biotecnologia Aplicada à Agropecuária (BIOAGRO) e no Viveiro de Pesquisas Florestais do Departamento de Engenharia Florestal da Universidade Federal de Viçosa, em Viçosa, MG.

A nutrição mineral utilizada na formação das mudas micropropagadas foi composta por superfosfato simples $\left(8 \mathrm{~kg} \mathrm{~m}^{-3}\right)$ em mistura com o substrato e pela nutrição complementar composta pela solução de sulfato de amônio $\left(20 \mathrm{~g} \mathrm{~L}^{-1}\right)$, cloreto de potássio $\left(3,33 \mathrm{~g} \mathrm{~L}^{-1}\right)$, sulfato de zinco $\left(0,22 \mathrm{~g} \mathrm{~L}^{-1}\right)$, sulfato de cobre $\left(0,22 \mathrm{~g} \mathrm{~L}^{-1}\right)$, sulfato de manganês $\left(0,22 \mathrm{~g} \mathrm{~L}^{-1}\right)$ e ácido bórico $\left(0,39 \mathrm{~g} \mathrm{~L}^{-1}\right)$, sendo aplicados $2,5 \mathrm{~mL}$ dessa solução em cada tubete, duas vezes por semana. Essas mudas foram transferidas para o viveiro da empresa, onde receberam a mesma adubação das mudas propagadas pelas outras técnicas (estaquia, miniestaquia e microestaquia), por um período de três semanas antes do plantio.

Para a formação das mudas por estaquia, microestaquia e miniestaquia foram usados, como recipientes, tubetes plásticos de $55 \mathrm{~cm}^{3}$ de capacidade contendo mistura de casca de arroz carbonizada (50\%) e vermiculita de granulometria média (50\%). A nutrição mineral utilizada na formação das mudas foi composta por superfosfato simples $\left(8 \mathrm{~kg} \mathrm{~m}^{-3}\right)$ em mistura com

R. Árvore, Viçosa-MG, v.30, n.4, p.503-512, 2006 
o substrato e pela nutrição complementar composta pela solução de sulfato de amônio $\left(18 \mathrm{~g} \mathrm{~L}^{-1}\right)$, cloreto de potássio $\left(3,33 \mathrm{~g} \mathrm{~L}^{-1}\right)$, sulfato de zinco $\left(0,22 \mathrm{~g} \mathrm{~L}^{-1}\right)$, sulfato de cobre $\left(0,22 \mathrm{~g} \mathrm{~L}^{-1}\right)$ e ácido bórico $\left(0,39 \mathrm{~g} \mathrm{~L}^{-1}\right)$, sendo aplicados $2,5 \mathrm{~mL}$ dessa solução por tubete ( 2 vezes por semana).

\subsection{Tratamentos e delineamento experimental}

Após a obtenção das mudas pelas técnicas de estaquia, micropropagação, microestaquia e miniestaquia, elas foram transferidas para o campo, onde foi instalado um teste clonal e preparada uma parcela experimental em cada região de estudo, com a denomição de "local 1" (Fazenda Corredor - Bocaiúva, MG) e "local 2" (Fazenda Campo Alegre - João Pinheiro, MG).

O teste clonal foi instalado seguindo o delineamento de blocos ao acaso, no esquema de parcela subdividida (clone e técnica de propagação), com oito repetições e parcelas lineares de quatro plantas, no espaçamento de 3 x 3 m, visando atender à necessidade de avaliações silviculturais de crescimento em altura e diâmetro das plantas. Paralelamente ao teste clonal, foi instalada uma "parcela experimental" constituída por parcelabase de " 6 plantas x 5 linhas" sem bordadura entre elas, num total de 30 plantas por técnica de propagação, por clone, segundo o esquema de amostragem seletiva, para atender à necessidade de avaliações destrutivas de planta referente à biomassa da parte aérea.

O experimento foi instalado em dezembro de 2000 , em uma área de reforma anteriormente ocupada por um povoamento de eucalipto. Em ambos os locais e experimentos, o solo foi preparado por meio de subsolagem, a uma profundidade de $50 \mathrm{~cm}$ em linha. No momento do plantio, o sistema radicular das mudas foi imerso em uma solução de $1,0 \%$ de fosfato monoamônio (MAP), recebendo posteriormente (entre 10 a 25 dias após o plantio) uma adubação suplementar de $100 \mathrm{~g} /$ planta de NPK (06-30-06) + 7\% S aplicada em coveta lateral.

\subsection{Avaliações}

As avaliações no teste clonal e na parcela experimental foram aos 4, 8, 16 e 24 meses de idade, a contar da data de implantação do experimento. No teste clonal, foram mensuradas as características de crescimento em altura e diâmetro a 1,30 m do solo (dap), de todos os indivíduos dentro de cada parcela, sendo o dap analisado a partir da terceira avaliação (16 e 24 meses de idade). Quanto à parcela experimental, foram feitas as avaliações de cunho destrutivos, em relação ao crescimento da parte aérea das árvores, obtendose a biomassa foliar dos galhos e do tronco a partir de três árvores médias representativas da parcela.

Para a obtenção da biomassa das folhas e dos galhos, as árvores abatidas foram desgalhadas rente ao tronco e desfolhadas. Em seguida, obteve a biomassa úmida e desta foi retirada uma amostra de $300 \mathrm{~g}$, a qual foi secada em estufa na temperatura de $70^{\circ} \mathrm{C}$ até o peso de matéria seca constante, obtendo-se o peso da matéria seca foliar e dos galhos.

Após ser desgalhado, o tronco foi dividido em três segmentos iguais em comprimento, e, após obter a biomassa úmida, retirou-se uma amostra de mesmo comprimento $(2 \mathrm{~cm})$ em cada seção, constituindo três amostras por tronco/árvore. Após conhecer o peso da matéria seca das amostras, obteve-se o peso da matéria seca do lenho, conforme procedimentos adotados para biomassa foliar e dos galhos.

Nas avaliações das características, aos 4 e 8 meses de idade, foram utilizados os dados obtidos por Santos (2003). Vale salientar que as avaliações de altura aos 8 meses não foram obtidas no local 1 , assim como os dados referentes à estaquia do clone VM1 no teste clonal e na parcela experimental. A técnica de microestaquia do clone VM1 e as técnicas de microestaquia e miniestaquia do clone VM2, na parcela experimental, também não foram incluídas no local 2 por motivos operacionais.

Os dados resultantes foram analisados por meio de análise de variância e testes de médias (teste de Tukey) a 5\% de probabilidade, utilizando-se o programa SAEG (Sistema para Análises Estatísticas), da Universidade Federal de Viçosa.

\section{RESULTADOS E DISCUSSÕES}

\subsection{Crescimento em altura}

No que tange ao crescimento em altura das plantas, avaliado aos 4, 8, 16 e 24 meses de idade, observouse, de modo geral, resposta similar entre as técnicas de propagação na maioria dos clones e nas diversas idades de avaliação (Figura 1). 


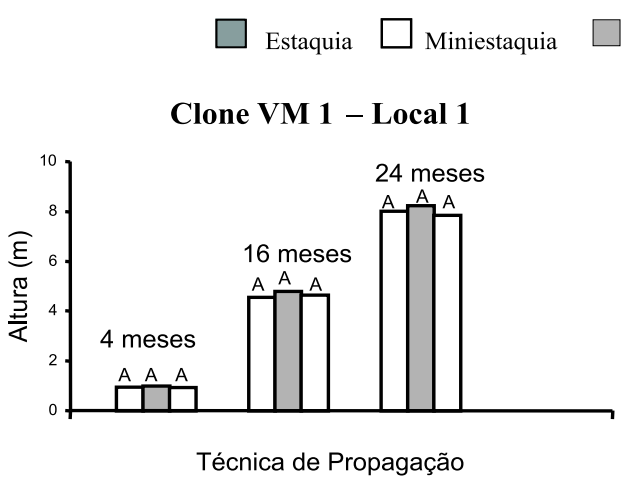

Microestaquia $\square$ Micropropagação

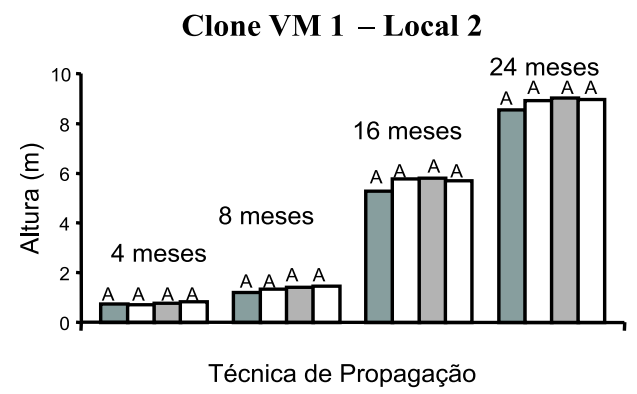

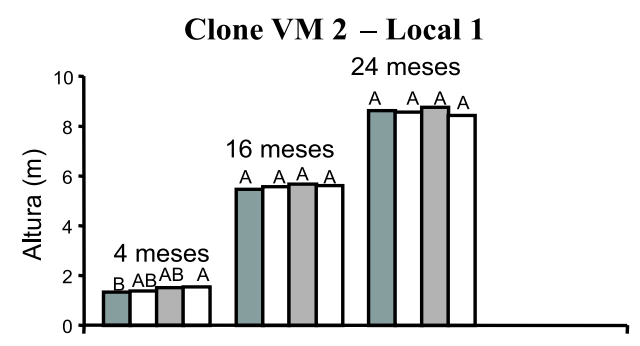

Técnica de Propagação

Clone VM 3 - Local 1

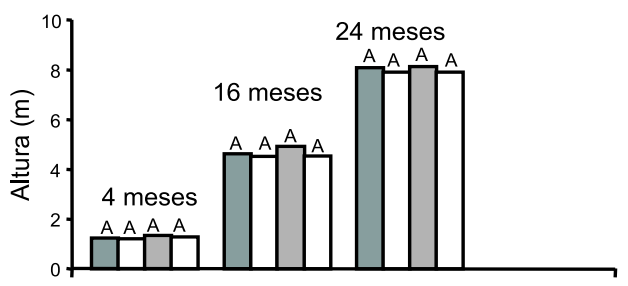

Técnica de Propagação

Clone VM 4 - Local 1

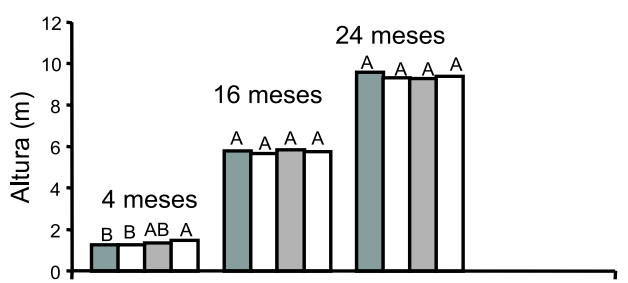

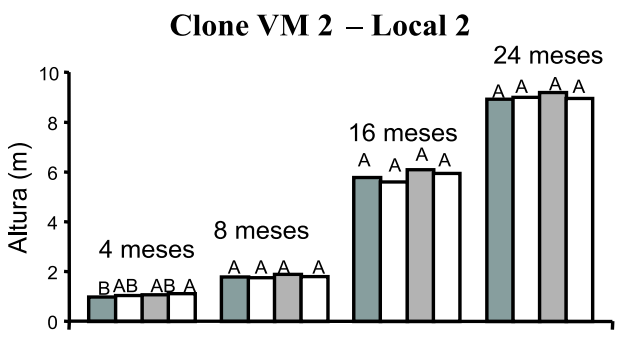

Técnica de Propagação

Clone VM 3 - Local 2

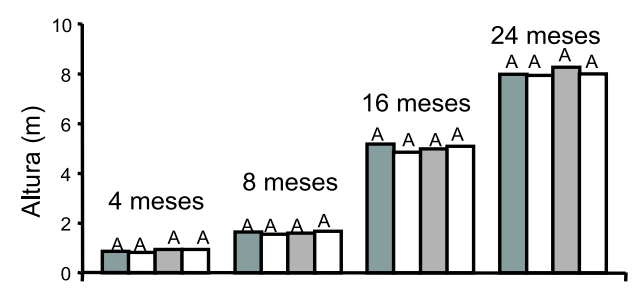

Técnica de Propagação

Clone VM 4 - Local 2

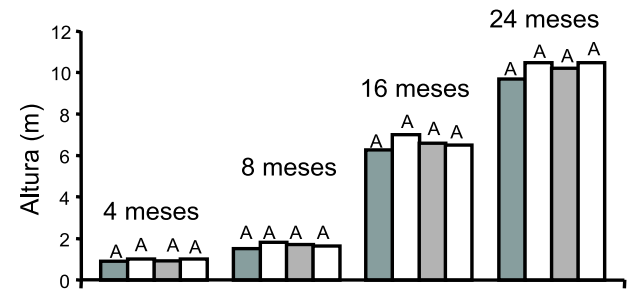

Obs.: Médias seguidas de uma mesma letra dentro de cada clone, local e idade entre as técnicas de propagação não diferem entre si, pelo teste de Tukey a $5 \%$ de probabilidade.

Figura 1 - Médias de crescimento em altura aos 4, 8, 16 e 24 meses de idade, em função da técnica de propagação dos quatro clones de Eucalyptus spp.

Figure 1 -Growth height means at 4, 8, 16 and 24 months of age, in relation to propagation techniques of four Eucalyptus spp. clones. 
As diferenças fisiológicas e ontogenéticas observadas entre os propágulos foram insuficientes para expressar diferenças em crescimento no campo. Espera-se que propágulos mais juvenis, como os usados na micropropagação e microestaquia, apresentem melhor desenvolvimento no campo, pois resultados superiores com o uso da micropropagação, em comparação com a macropropagação, foram obtidos por Bell et al. (1993), Denison e Kietzka (1993), Rockwood e Warrag (1994), Watt et al. (1995) e Xavier et al. (1997).

Exceção ocorreu nos clones VM2 e VM4, em fase inicial do teste clonal, aos 4 meses, que apresentaram diferenças significativas entre as técnicas de propagação. Em relação ao clone VM2, a micropropagação foi significativamente superior em relação à estaquia nos dois locais em razão, possivelmente, da utilização de material vegetativo, rejuvenescido in vitro, que tendem a promover a minimização do efeito " $C$ ". A redução desse efeito, neste caso, além do rejuvenescimento in vitro, pode ser atribuída ao estado fisiológico dos propágulos vegetativos, pois as mudas propagadas pelas técnicas de miniestaquia, microestaquia e micropropagação foram provenientes de propágulos vegetativos herbáceos e apicais e aquelas propagadas por estaquia foram confeccionadas a partir de estacas semilenhosas e intermediárias. Em relação ao clone VM4, observaram-se, aos 4 meses de idade, resultados distintos em relação ao local de plantio das mudas. No local 1, verificou-se melhor "performance" com o uso da micropropagação e microestaquia em relação à miniestaquia e estaquia. No entanto, no local 2 constatou-se a não-ocorrência de diferenças significativas entre as técnicas de propagação. Em trabalho realizado por Xavier et al. (2001) utilizando material genético VM2 e VM4, observou-se que o primeiro demonstrou maior aptidão à propagação, no entanto, em ambos os clones, a microestaquia proporcionou condições de viabilidade na propagação clonal, em função dos altos porcentuais de enraizamento. Esses mesmos autores ressaltaram ainda que o clone de menor aptidão para a propagação clonal (VM4) apresentou melhor resposta para a microestaquia, resultando na suposição de que material genético menos propenso à propagação clonal daria melhores respostas ao rejuvenescimento in vitro.

Assim, com base nos resultados deste estudo, infere-se para alguns clones com menor propensão à propagação vegetativa que o uso de propágulos vegetativos mais juvenis pode proporcionar maior crescimento em altura na fase inicial de crescimento no campo, a exemplo de VM2 e VM4 aos 4 meses de idade, devido ao seu maior vigor inicial. Entretanto, esse crescimento não foi evidenciado a partir de 8 meses de idade. Vale ressaltar que para clones com facilidade na propagação clonal as técnicas de estaquia, micropropagação, microestaquia e miniestaquia apresentaram desempenho silvicultural semelhante.

\subsection{Crescimento em diâmetro (dap)}

Baseando-se nos preceitos teóricos sobre juvenilidade e maturação, em que é definido que plantas mais juvenis tendem a possuir maior vigor vegetativo e maior tendência ao crescimento vegetativo (GREENWOOD e HUTCHISON, 1993; HARTMANN et al., 1997), seria esperado que técnicas que utilizam propágulos mais juvenis como a micropropagação e microestaquia apresentassem maior crescimento em diâmetro. Porém, com base na Figura 2, não se observou diferença significativa entre as técnicas, indicando que as respostas fisiológicas e ontogenéticas entre os propágulos foram similares na expressão do comportamento de crescimento em diâmetro (dap), avaliado aos 16 e 24 meses, nos dois locais.

Cabe ressaltar que os efeitos da maturação se expressam ao longo do ciclo de vida das plantas. Dessa forma, considerando que os clones neste estudo foram provenientes de árvores selecionadas aos 7 anos de idade, os efeitos da maturação sobre as características avaliadas não foram acentuados o suficiente para evidenciar diferenças no seu desempenho silvicultural. Entretanto, segundo Hackett e Murray (1993), as características relacionadas à maturação são reversíveis, porém não apresentam o mesmo grau de facilidade na reversão, que é, para determinada característica, variável em decorrência do grau de juvenilidade do material.

\subsection{Biomassa da parte aérea}

De acordo com os dados apresentados nos Quadros 1 e 2, verificaram-se para o peso da matéria seca foliar e dos galhos respostas variadas em relação às técnicas de propagação, avaliadas aos 4, 8, 16 e 24 meses de idade nos dois locais. Todavia, não se observou resposta consistente nas características estudadas como resultado dos tratamentos aplicados. Os coeficientes de variação experimental obtidos com relação a essas características variaram de 16 a $31 \%$ no local 1 e de 30 a $38 \%$ no local 2 . 
Estaquia $\square$ Miniestaquia $\square$ Microestaquia $\Xi_{\substack{\text { Micropropagaçã } \\ \text { M }}}$

Clone VM 1 - Local 1

Clone VM 1 - Local 2

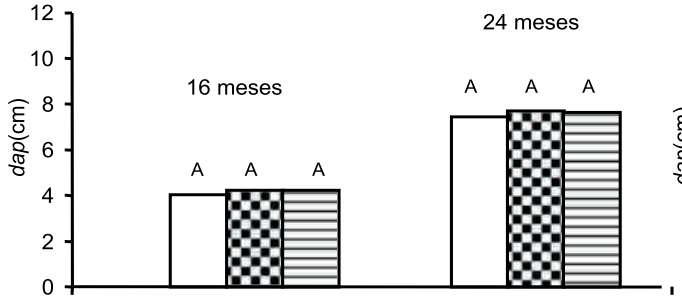

Técnica de Propagação

Clone VM 2 - Local 1

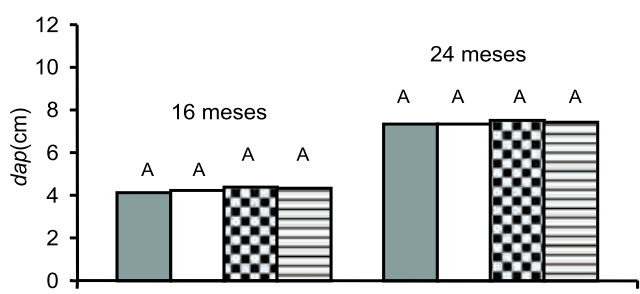

Técnica de Propagação

Clone VM 3 - Local 1

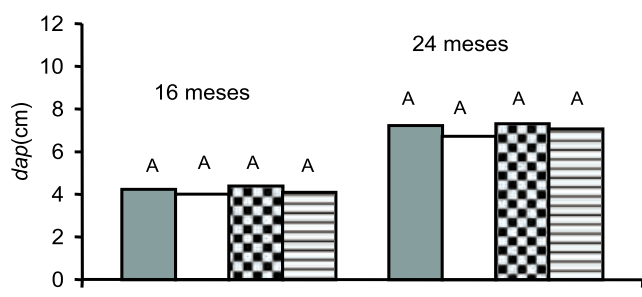

Técnica de Propagação

Clone VM 4 - Local 1

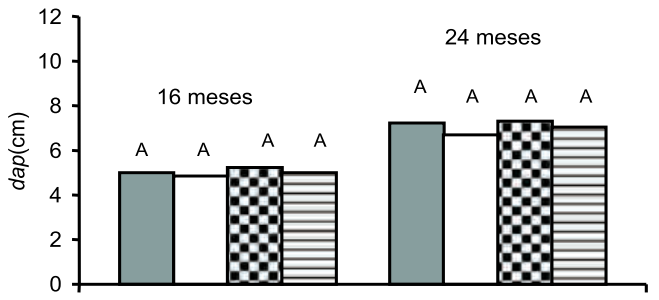

Técnica de Propagação

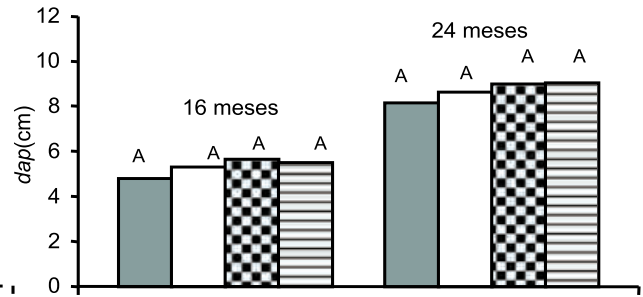

Técnica de Propagação

Clone VM 2 - Local 2

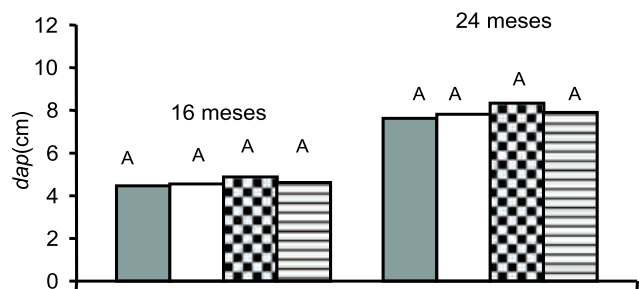

Técnica de Propagação

Clone VM 3 - Local 2

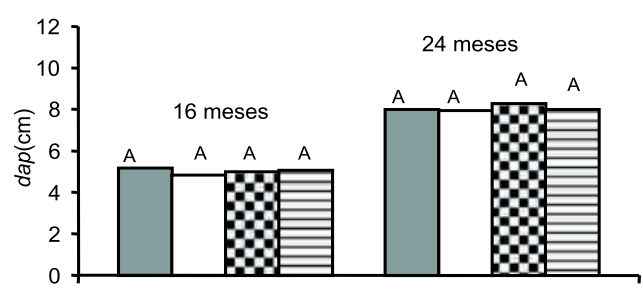

Técnica de Propagação

Clone VM 4 - Local 2

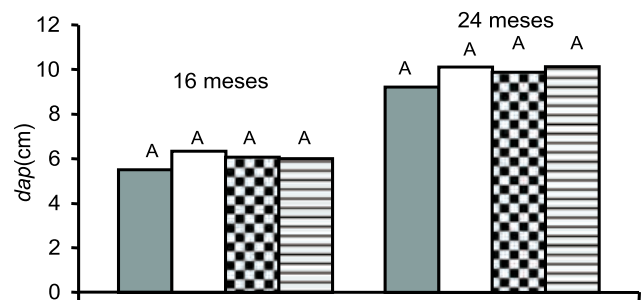

Técnica de Propagação

Obs.: Médias seguidas de uma mesma letra dentro de cada clone, idade e local entre as técnicas de propagação não diferem entre si, pelo teste de Tukey a $5 \%$ de probabilidade.

Figura 2 - Médias do crescimento em diâmetro (dap), aos 16 e 24 meses, em função da técnica de propagação dos quatro clones de Eucalyptus spp.

Figure 2 - Growth diameter (dap) means at 16 and 24 months of age, in relation to propagation techniques offour Eucalyptus spp. clones. 
Quadro 1 - Médias do peso de matéria seca da parte aérea aos 4, 8, 16 e 24 meses de idade, em Bocaiúva (MG), em função da técnica de propagação e dos quatro clones de Eucalyptus spp.

Table 1 -Means of aerial part dry matter weight at 4, 8, 16 and 24 months of age, at site 1, in relation to propagation techniques of four Eucalyptus spp. clones

\begin{tabular}{|c|c|c|c|c|c|c|c|c|c|c|c|c|c|c|c|c|c|}
\hline \multirow[b]{2}{*}{ Clone } & \multirow[b]{2}{*}{$\begin{array}{l}\text { Técnica de } \\
\text { Propagação }\end{array}$} & \multicolumn{16}{|c|}{ Peso de Matéria Seca da Parte Aérea (kg) } \\
\hline & & \multicolumn{4}{|c|}{$\begin{array}{c}\text { Folha } \\
\text { Idade (meses) }\end{array}$} & \multicolumn{4}{|c|}{$\begin{array}{c}\text { Galho } \\
\text { Idade (meses) }\end{array}$} & \multicolumn{4}{|c|}{$\begin{array}{c}\text { Lenho } \\
\text { Idade (meses) } \\
\end{array}$} & \multicolumn{4}{|c|}{$\begin{array}{c}\text { Total } \\
\text { Idade (meses) }\end{array}$} \\
\hline \multirow[t]{2}{*}{ VM1 } & T1 & & & & & & & & & & & & & & & & \\
\hline & $\mathrm{T} 4$ & $0,08 \mathrm{a}$ & $0,12 \mathrm{~b}$ & $1,35 \mathrm{a}$ & 3,89 a & $0,02 \mathrm{a}$ & $0,06 \mathrm{~b}$ & $0,69 \mathrm{a}$ & 2,26 a & $0,02 \mathrm{a}$ & 0,07 a & $1,33 \mathrm{a}$ & 5,46 a & 0,07 a & $0,25 \mathrm{a}$ & $3,38 \mathrm{a}$ & 11,6 a \\
\hline \multirow[t]{3}{*}{ VM2 } & $\mathrm{T} 1$ & $0,074 \mathrm{~b}$ & $0,12 \mathrm{~b}$ & $1,43 \mathrm{a}$ & $3,32 \mathrm{~b}$ & $0,03 \mathrm{~b}$ & $0,07 \mathrm{a}$ & $1,26 \mathrm{~b}$ & $2,26 \mathrm{c}$ & $0,03 \mathrm{~b}$ & $0,07 \mathrm{a}$ & $1,46 \mathrm{~b}$ & & $0,07 \mathrm{~b}$ & $0,26 \mathrm{a}$ & & \\
\hline & $\mathrm{T} 2$ & $0,07 \mathrm{~b}$ & $0,14 a b$ & $2,09 \mathrm{a}$ & 3,96 a & $0,03 \mathrm{~b}$ & $0,08 \mathrm{a}$ & $1,83 \mathrm{a}$ & $3,65 \mathrm{ab}$ & $0,02 \mathrm{~b}$ & $0,08 \mathrm{a}$ & $1,98 \mathrm{ab}$ & $6,52 \mathrm{a}$ & $0,08 \mathrm{~b}$ & $0,31 \mathrm{a}$ & $5,91 \mathrm{ab}$ & 14,10 \\
\hline & T3 & $0,17 \mathrm{a}$ & $0,17 \mathrm{a}$ & $1,94 \mathrm{a}$ & 4,69 a & $0,06 \mathrm{a}$ & $0,09 \mathrm{a}$ & $1,80 \mathrm{ab}$ & 5,01 a & $0,05 \mathrm{a}$ & $0,10 \mathrm{a}$ & $2,70 \mathrm{a}$ & 8,17 a & $0,17 \mathrm{a}$ & $0,36 \mathrm{a}$ & $6,45 \mathrm{a}$ & 17,90 \\
\hline & $\mathrm{T} 3$ & $0,08 \mathrm{a}$ & $0,09 \mathrm{a}$ & $1,50 \mathrm{a}$ & $2,76 \mathrm{a}$ & $0,03 \mathrm{a}$ & $0,05 \mathrm{a}$ & $0,56 \mathrm{a}$ & $1,65 \mathrm{ab}$ & $0,05 \mathrm{a}$ & $0,12 \mathrm{a}$ & $1,60 \mathrm{ab}$ & $9,03 \mathrm{a}$ & $0,08 \mathrm{a}$ & $0,26 \mathrm{a}$ & $3,65 \mathrm{a}$ & $13,40 \mathrm{ab}$ \\
\hline & $\mathrm{T} 4$ & $0,07 \mathrm{ab}$ & $0,10 \mathrm{a}$ & $1,25 \mathrm{a}$ & $2,74 \mathrm{a}$ & $0,02 \mathrm{a}$ & $0,06 \mathrm{a}$ & $0,74 \mathrm{a}$ & $2,07 \mathrm{a}$ & $0,04 \mathrm{ab}$ & $0,10 \mathrm{ab}$ & $1,89 \mathrm{a}$ & $9,82 \mathrm{a}$ & $0,07 \mathrm{ab}$ & $0,26 \mathrm{a}$ & $3,89 \mathrm{a}$ & $14,60 \mathrm{a}$ \\
\hline \multirow[t]{4}{*}{ VM4 } & $\mathrm{T} 1$ & $0,08 \mathrm{a}$ & $0,15 \mathrm{a}$ & $1,12 \mathrm{a}$ & $3,91 \mathrm{a}$ & $0,03 \mathrm{a}$ & $0,09 \mathrm{a}$ & $0,75 \mathrm{a}$ & $2,79 \mathrm{a}$ & $0,03 \mathrm{bc}$ & $0,13 a b$ & $1,32 \mathrm{ab}$ & & $0,08 \mathrm{a}$ & $0,36 \mathrm{a}$ & $3,19 \mathrm{a}$ & $16,40 \mathrm{a}$ \\
\hline & $\mathrm{T} 2$ & $06 \mathrm{a}$ & $0,10 \mathrm{a}$ & $1,13 \mathrm{a}$ & $3,75 \mathrm{a}$ & $0,02 \mathrm{a}$ & $0,08 \mathrm{a}$ & $0,73 \mathrm{a}$ & $2,64 \mathrm{a}$ & $0,03 \mathrm{c}$ & $0,10 \mathrm{~b}$ & $1,16 \mathrm{~b}$ & 8,8 & 0,0 & $0,30 \mathrm{a}$ & $3,01 \mathrm{a}$ & $15,2 \mathrm{a}$ \\
\hline & T3 & $0,08 \mathrm{a}$ & $0,16 \mathrm{a}$ & $1,76 \mathrm{a}$ & $3,95 \mathrm{a}$ & $0,04 \mathrm{a}$ & $0,09 \mathrm{a}$ & $0,91 \mathrm{a}$ & $2,87 \mathrm{a}$ & $0,05 a b$ & $0,15 \mathrm{a}$ & $2,22 \mathrm{a}$ & $10,57 \mathrm{a}$ & $0,09 \mathrm{a}$ & $0,40 \mathrm{a}$ & $4,89 \mathrm{a}$ & $17,30 \mathrm{a}$ \\
\hline & $\mathrm{T} 4$ & $0,09 \mathrm{a}$ & $0,14 \mathrm{a}$ & $1,35 \mathrm{a}$ & $3,87 \mathrm{a}$ & $0,04 \mathrm{a}$ & $0,07 \mathrm{a}$ & $0,89 \mathrm{a}$ & $2,93 \mathrm{a}$ & $0,05 \mathrm{a}$ & $0,12 \mathrm{ab}$ & $2,12 \mathrm{a}$ & $10,3 \mathrm{a}$ & $0,09 \mathrm{a}$ & $0,32 \mathrm{a}$ & $4,37 \mathrm{a}$ & $17,10 \mathrm{a}$ \\
\hline
\end{tabular}

Obs. 1: $\mathrm{T} 1=$ estaquia, $\mathrm{T} 2=$ miniestaquia, $\mathrm{T} 3=$ microestaquia e $\mathrm{T} 4=$ micropropagação. Obs. 2: Médias seguidas de uma mesma letra dentro de cada clone, característica e idade entre as técnicas de propagação para cada idade não diferem entre si, pelo teste de Tukey a $5 \%$ de probabilidade.

Quadro 2 - Médias do peso de matéria seca da parte aérea aos 4, 8, 16 e 24 meses de idade, em João Pinheiro (MG), em função da técnica de propagação dos quatro clones de Eucalyptus spp.

Table 2 - Means of aerial part dry matter weight at 4, 8, 16 and 24 months of age, at site 2, in relation to propagation techniques of four Eucalyptus spp. clones

\begin{tabular}{|c|c|c|c|c|c|c|c|c|c|c|c|c|c|c|c|c|c|}
\hline \multirow{3}{*}{ Clone } & \multirow{3}{*}{$\begin{array}{l}\text { Técnica de } \\
\text { Propagação }\end{array}$} & & & & & & & & & & & & & & & & \\
\hline & & \multicolumn{4}{|c|}{$\begin{array}{c}\text { Folha } \\
\text { Idade (meses) }\end{array}$} & \multicolumn{4}{|c|}{$\begin{array}{c}\text { Galho } \\
\text { Idade (meses) }\end{array}$} & \multicolumn{4}{|c|}{$\begin{array}{c}\text { Lenho } \\
\text { Idade (meses) }\end{array}$} & \multicolumn{4}{|c|}{$\begin{array}{c}\text { Total } \\
\text { Idade (meses) }\end{array}$} \\
\hline & & 4 & 8 & 16 & 24 & 4 & 8 & 16 & 24 & 4 & 8 & 16 & 24 & 4 & 8 & 16 & 24 \\
\hline \multirow[t]{3}{*}{$\begin{array}{l}\text { VM1 } \\
\end{array}$} & $\mathrm{T} 1$ & $0,03 \mathrm{a}$ & $0,17 \mathrm{a}$ & $2,10 \mathrm{~b}$ & $5,55 \mathrm{a}$ & $0,01 \mathrm{~b}$ & $0,05 \mathrm{~b}$ & $1,04 \mathrm{~b}$ & $4,39 \mathrm{ab}$ & $0,07 \mathrm{a}$ & $0,06 \mathrm{~b}$ & $1,28 \mathrm{~b}$ & $7,44 \mathrm{~b}$ & $0,05 \mathrm{~b}$ & $0,29 \mathrm{a}$ & $4,42 b$ & $17,40 \mathrm{~b}$ \\
\hline & $\begin{array}{l}\text { T2 } \\
\text { T3 }\end{array}$ & 0,06 a & 0,18 a & $2,51 \mathrm{a}$ & 7,07 a & $0,02^{\mathrm{a}}$ & $0,06 \mathrm{~b}$ & $1,30 \mathrm{ab}$ & $3,83 \mathrm{~b}$ & $0,014 \mathrm{a}$ & $0,05 \mathrm{~b}$ & $1,57 \mathrm{~b}$ & $9,51 \mathrm{~b}$ & $0,09 \mathrm{a}$ & $0,30 \mathrm{a}$ & $5,38 \mathrm{~b}$ & $20,40 \mathrm{~b}$ \\
\hline & $\mathrm{T} 4$ & $0,05 \mathrm{a}$ & $0,32 \mathrm{a}$ & $3,33 \mathrm{a}$ & 8,16 a & $0,01 \mathrm{ab}$ & $0,13 \mathrm{a}$ & $1,88 \mathrm{a}$ & $6,67 \mathrm{a}$ & $0,01 \mathrm{a}$ & $0,12 \mathrm{a}$ & $3,30 \mathrm{a}$ & $16,80 \mathrm{a}$ & $0,07 \mathrm{a}$ & $0,14 \mathrm{~b}$ & $8,50 \mathrm{a}$ & $31,60 \mathrm{a}$ \\
\hline \multirow[t]{4}{*}{ VM2 } & $\mathrm{T} 1$ & $0,02 \mathrm{~b}$ & $0,11 \mathrm{a}$ & $1,80 \mathrm{a}$ & $4,21 \mathrm{a}$ & $0,00 \mathrm{a}$ & $0,04 \mathrm{~b}$ & 1,36 a & $4,23 \mathrm{a}$ & $0,01 \mathrm{~b}$ & $0,05 \mathrm{a}$ & $1,58 \mathrm{a}$ & $7,66 \mathrm{a}$ & $0,03 \mathrm{~b}$ & $0,19 \mathrm{~b}$ & $4,75 \mathrm{a}$ & $16,10 \mathrm{a}$ \\
\hline & $\mathrm{T} 2$ & $0,04 \mathrm{a}$ & $0,15 \mathrm{a}$ & $1,96 \mathrm{a}$ & $5,38 \mathrm{a}$ & $0,01 \mathrm{a}$ & $0,07 \mathrm{a}$ & $1,84 \mathrm{a}$ & $5,49 \mathrm{a}$ & $0,02 \mathrm{a}$ & $0,07 \mathrm{a}$ & $1,88 \mathrm{a}$ & $8,68 \mathrm{a}$ & $0,08 \mathrm{a}$ & $0,28 \mathrm{a}$ & $5,67 \mathrm{a}$ & $19,60 \mathrm{a}$ \\
\hline & T3 & - & - & - & - & - & - & - & - & - & - & - & - & - & - & - & - \\
\hline & $\mathrm{T} 4$ & - & - & - & & - & - & & & - & & & - & & & & \\
\hline \multirow[t]{4}{*}{ VM3 } & $\mathrm{T} 1$ & $0,06 a$ & $0,23 \mathrm{a}$ & $1,51 \mathrm{a}$ & $2,49 \mathrm{a}$ & $0,02 \mathrm{a}$ & $0,07 \mathrm{a}$ & 0,66 a & $1,62 \mathrm{a}$ & $0,03 \mathrm{a}$ & $0,18 \mathrm{a}$ & $2,07 \mathrm{~b}$ & 8,48 a & $0,10 \mathrm{a}$ & $0,48 \mathrm{a}$ & $4,24 \mathrm{~b}$ & $12,58 \mathrm{a}$ \\
\hline & $\mathrm{T} 2$ & $0,06 \mathrm{a}$ & $0,18 \mathrm{ab}$ & $1,71 \mathrm{a}$ & $2,74 \mathrm{a}$ & $0,02^{\mathrm{a}}$ & $0,07 \mathrm{a}$ & $0,80 \mathrm{a}$ & $1,59 \mathrm{a}$ & $0,03 \mathrm{a}$ & $0,15 \mathrm{a}$ & $3,54 \mathrm{ab}$ & $9,66 \mathrm{a}$ & $0,11 \mathrm{a}$ & $0,40 \mathrm{a}$ & $6,05 \mathrm{ab}$ & $13,98 \mathrm{a}$ \\
\hline & T3 & $0,04 \mathrm{a}$ & $0,14 \mathrm{~b}$ & $2,42 \mathrm{a}$ & $3,79 \mathrm{a}$ & $0,01 \mathrm{a}$ & $0,05 \mathrm{a}$ & $1,26 \mathrm{a}$ & $2,01 \mathrm{a}$ & $0,02 \mathrm{a}$ & $0,12 \mathrm{a}$ & $4,78 \mathrm{a}$ & $12,36 \mathrm{a}$ & $0,07 \mathrm{a}$ & $0,32 \mathrm{a}$ & $8,45 \mathrm{a}$ & $18,16 \mathrm{a}$ \\
\hline & $\mathrm{T} 4$ & $0,05 \mathrm{a}$ & $0,15 \mathrm{~b}$ & $2,23 \mathrm{a}$ & $2,92 \mathrm{a}$ & $0,02^{\mathrm{a}}$ & $0,06 \mathrm{a}$ & $1,12 \mathrm{a}$ & $1,75 \mathrm{a}$ & $0,03 \mathrm{a}$ & $0,15 \mathrm{a}$ & $4,66 \mathrm{a}$ & $9,56 \mathrm{a}$ & $0,10 \mathrm{a}$ & $0,36 \mathrm{a}$ & $8,02 \mathrm{a}$ & $14,24 \mathrm{a}$ \\
\hline \multirow[t]{4}{*}{ VM4 } & $\mathrm{T} 1$ & $0,08 \mathrm{a}$ & $0,28 \mathrm{a}$ & $2,58 \mathrm{a}$ & $4,50 \mathrm{a}$ & $0,03 \mathrm{a}$ & 0,16 a & $1,63 \mathrm{a}$ & $4,34 \mathrm{a}$ & $0,03 \mathrm{a}$ & $0,18 \mathrm{a}$ & $3,98 \mathrm{a}$ & $11,48 \mathrm{ab}$ & $0,13 \mathrm{a}$ & $0,62 \mathrm{a}$ & $8,20 \mathrm{a}$ & $24,30 \mathrm{a}$ \\
\hline & $\mathrm{T} 2$ & $0,05 \mathrm{a}$ & $0,21 \mathrm{a}$ & $1,98 \mathrm{a}$ & $4,06 \mathrm{a}$ & $0,01 \mathrm{a}$ & $0,09 \mathrm{ab}$ & $1,55 \mathrm{a}$ & $2,63 \mathrm{a}$ & $0,02 \mathrm{a}$ & $0,13 \mathrm{a}$ & $2,11 \mathrm{~b}$ & $15,46 \mathrm{~b}$ & $0,09 \mathrm{a}$ & $0,43 \mathrm{a}$ & $5,65 \mathrm{a}$ & $18,30 \mathrm{a}$ \\
\hline & T3 & $0,06 \mathrm{a}$ & $0,18 \mathrm{a}$ & $2,30 \mathrm{a}$ & $4,82 \mathrm{a}$ & $0,02 \mathrm{a}$ & $0,07 \mathrm{~b}$ & $1,55 \mathrm{a}$ & 3,93 a & $0,03 \mathrm{a}$ & $0,10 \mathrm{a}$ & $3,71 \mathrm{a}$ & $15,99 \mathrm{ab}$ & $0,11 \mathrm{a}$ & $0,35 \mathrm{a}$ & $7,56 \mathrm{a}$ & $24,70 \mathrm{a}$ \\
\hline & $\mathrm{T} 4$ & $0,04 \mathrm{a}$ & $0,20 \mathrm{a}$ & $2,06 \mathrm{a}$ & 5,79 a & $0,01 \mathrm{a}$ & $0,10 a b$ & $1,50 \mathrm{a}$ & $4,10 \mathrm{a}$ & $0,02 \mathrm{a}$ & $0,13 \mathrm{a}$ & $3,63 \mathrm{ab}$ & 18,01 a & $0,08 \mathrm{a}$ & $0,43 \mathrm{a}$ & $7,19 \mathrm{a}$ & $27,90 \mathrm{a}$ \\
\hline
\end{tabular}

Obs. 1: $\mathrm{T} 1=$ estaquia, $\mathrm{T} 2=$ miniestaquia, $\mathrm{T} 3=$ microestaquia e $\mathrm{T} 4=$ micropropagação. Obs. 2 : Médias seguidas de uma mesma letra dentro de cada clone, característica e idade entre as técnicas de propagação para cada idade não diferem entre si, pelo teste de Tukey a $5 \%$ de probabilidade. 
Quanto ao peso da matéria seca foliar, no clone VM4, por exemplo, observou-se a não-ocorrência de diferenças significativas entre as técnicas de propagação, nas quatro idades e nos dois locais. Isso indica que a diferença de juvenilidade entre os propágulos vegetativos foi insuficiente para expressar resposta diferenciada. No entanto, verificaram-se menores pesos com o uso da micropropagação em comparação com a microestaquia para VM1 e VM2 aos 8 meses de idade, bem como resultados superiores com a estaquia em comparação com a miniestaquia para o clone VM3 aos 8 meses. Assim, notou-se uma resposta não consistente, apesar de serem escassos os registros na literatura sobre avaliação da influência de técnicas de propagação clonal no peso da matéria seca foliar. Tanto no local 2 (Quadro 2), quanto no 1 (Quadro 1), observou-se diversidade de resposta em relação às técnicas de propagação, épocas de avaliação e material genético utilizado. De modo geral, pode-se dizer que as respostas se apresentaram inconsistentes.

Quando se avaliou a biomossa dos galhos, observaram-se diferenças significativas entre as técnicas de propagação. No clone VM2 aos 24 meses, no local 1 , a microestaquia mostrou-se superior à estaquia, com possíveis efeitos do uso de propagulos mais juvenis. Entretanto, independentemente do local e em algumas idades de avaliação, existem respostas superiores com o uso da estaquia, em comparação com a miniestaquia, para o clone VM3 aos 8 meses de idade.

Segundo Wendling (2002), a possibilidade de ocorrerem influências negativas dos métodos de rejuvenescimento em clones com maior grau de juvenilidade está associada ao fato de existir uma linha de máxima juvenilidade. A partir dessa linha, qualquer método ou técnica de rejuvenescimento não mais resultaria em respostas positivas, ocasionando, em algumas situações, redução no vigor geral dos propágulos, em razão dos tratamentos e manejo envolvidos.

Em alguns clones e em determinadas idades, as técnicas, a princípio, com maior grau de juvenilidade proporcionaram maior alocação de biomassa para o lenho. No local 1 aos 16 meses, a microestaquia no clone VM2 apresentou melhores resultados do que a técnica de estaquia. Melhores resultados foram observados com a microestaquia, nos clones VM3 e VM4 aos 4 e 8 meses, em comparação com a miniestaquia.
Resultados superiores foram obtidos com a micropropagação e microestaquia no clone VM3 aos 24 meses, em comparação com a miniestaquia e estaquia, e superiores à miniestaquia no clone VM4 aos 16 meses. No entanto, de modo geral o uso de propágulos vegetativos mais juvenis para a biomassa das folhas e galhos não apresentou resposta positiva.

\section{CONCLUSÕES}

De acordo com os resultados e nas condições em que foram instalados os experimentos, de forma geral pode-se concluir que os efeitos das técnicas de propagação não resultaram em diferenças significativas no crescimento em altura e dap, nas idades avaliadas, dos clones estudados. Em relação à biomassa da parte aérea, os resultados, de modo geral, não apresentaram efeitos das técnicas de propagação em relação ao comportamento silvicultural dos clones.

Maior número de subcultivos in vitro pela micropropagação seriada, assim como a utilização de clones com maior dificuldade de propagação clonal e selecionados a partir de árvores em idades mais avançadas, pode resultar em efeitos mais pronunciados no rejuvenescimento.

\section{AGRADECIMENTOS}

À Empresa V \& M Florestal, pela disponibilização do material experimental (clones) e pelo apoio orçamentário e estrutural na condução da pesquisa; e ao CNPq (Conselho Nacional de Pesquisa e Desenvolvimento Científico e Tecnológico), pelo suporte financeiro.

\section{REFERÊNCIAS BIBLIOGRÁFICAS}

ASSIS, T. F. Propagação vegetativa de Eucalyptus por microestaquia. In: IUFRO CONFERENCE ON SILVICULTURE AND IMPROVEMENT OF EUCALYPTS, 1997, Salvador. Proceedings... Colombo: EMBRAPA, 1997. v. 1, p. 300-304.

BELL, D. T. et al. Comparisons of growth of Eucalyptus camaldulensis from seeds and tissue culture: root, shoot and leaf morphology of 9- month - old plants grown in deep sand and sand over clay. Forest Ecology and Management, v. 57, p. 125-139, 1993.

R. Árvore, Viçosa-MG, v.30, n.4, p.503-512, 2006 
DENISON, N. P.; KIETZKA, J. E. The development and utilization of vegetative propagation in Mondi for commercial afforestation programmes. Suid Afrikaanse Bobouthkrif. South African Forestry Journal, n. 166, p. 53-60, 1993.

FRAMPTON, L.J.; FOSTER, G.S. Field testing vegetative propagules. In: AHUJA, M. R.; LIBBY, W. J. (Ed.). Clonal forestry: genetics and biotechnology. Budapest: Springer-Verlag, 1993. p. 110-134.

GREENWOOD, M. S.; HUTCHISON, K. W. Maturation as an developmental process. In: AHUJA, M. R.; LIBBY, W. J. (Ed.). Clonal forestry: genetics and biotechnology. Budapest: Springer-Verlag, 1993. p. 14-33.

HACKETT, W. P.; MURRAY, J. R. Maturation and rejuvenation in woody species. In: AHUJA, M. R. (Ed.) Micropropagation of woody plants. Dordrecht: Kluwer Academic Publishers, 1993. p. 93-105.

HARTMANN, H. T.; KESTER, D. E.; DAVIES JUNIOR, F. T.; GENEVE, R. L. Plant propagation: principles and practices. 6.ed. New Jersey: Prentice-Hall, 1997. 770 p.

ROCKWOOD, D. L. et al. Field performance of micropropagated, macropropagated, and seed-derived propagules of three Eucalyptus grandis ortets. Plant Cell Reports., v. 13, p. 628-631, 1994.

SANTOS, A. P. Avaliação silvicultural de clones de Eucalyptus spp. propagados por macro e micropropagação. 2003. 51 f. Dissertação (Mestrado em Ciência Florestal) - Universidade Federal de Viçosa, Viçosa, 2003.
TITON, M. Propagação clonal de Eucalyptus grandis por miniestaquia e microestaquia. 2001. 65 f. Dissertação (Mestrado em Ciência Florestal) - Universidade Federal de Viçosa, Viçosa, 2001.

WATT, M. P.; DUNCAN, M. Ing.; BLAKEWAY, F. C.; HERMAN, B. Field performance of micropropagated Eucalyptus hybrids. South African Forestry Journal, n. 173, p. 17-21, 1995.

WENDLING, I. Rejuvenescimento de clones de Eucalyptus grandis por miniestaquia seriada e micropropagação. 2002. 99 f. Tese (Doutorado em Ciência Florestal) Universidade Federal de Viçosa, Viçosa, 2002.

XAVIER, A. et al. Desempenho do enraizamento de microestacas e miniestacas de clones de híbrido de Eucalyptus grandis. Revista Árvore, v. 25, n. 4, p. 403-411, 2001.

XAVIER, A.; COMÉRIO, J. Microestaquia: uma maximização da micropropagação de Eucalyptus. Revista Árvore, v. 20, n. 1, p. 9-16, 1996.

XAVIER, A.; COMÉRIO, J.; IANNELLI, C. M. Eficiência da estaquia, da microestaquia e da micropropagação na clonagem de Eucalyptus spp. In: IUFRO CONFERENCE ON SILVICULTURE AND IMPROVEMENT OF Eucalyptus. Proceedings... Colombo: EMBRAPA, 1997. v. 2. p. 40-45. 